\title{
Fuzziness in semantic memory: Choosing exemplars of subjective categories
}

\author{
GREGG C. ODEN \\ University of Wisconsin, Madison, Wisconsin 53706
}

\begin{abstract}
Class membership is a fundamental relationship between concepts in semantic memory. Recent research indicates that class membership may subjectively be a continuous type of relationship. The processing of information about the degree to which items belong to a particular class was investigated in an experiment in which subjects compared two statements describing class membership relationships. The results strongly supported a simple model which describes the judgment process as directly involving subjective degree-of-truthfulness values. The success of the model indicates that the subjects were able to process this kind of fuzzy information in a consistent and systematic manner. Some of the implications of the human competancy for processing fuzzy information are discussed.
\end{abstract}

Class membership has traditionally been treated as if it were a discrete all-or-none relationship. This has simplified the development and use of formal models of logical reasoning and has consequently also been adopted as a "convenient fiction," as Lakoff (1972) terms it, for psychological models of semantic memory. The price of this convenience is that we are relatively ignorant of the manner in which people use and cope with information about degrees of class membership. Recently, however, there has been considerable interest in studying this neglected topic, both in psychology and in linguistics, stimulated in part by the development over the past decade of logical models for dealing with "fuzzy" information (e.g., Zadeh, 1965, 1975).

Fuzzy set theory is a generalization of traditional discrete set theory that conceptualizes sets as having fuzzy rather than distinct boundaries. In such a conceptualization, many items are neither clearly members of the set nor clearly not members of the set. For example, while it is true that a bathtub is "sort of" furniture, it is certainly not as correct to say that a bathtub is furniture as it is to say that a table is furniture. This suggests another useful way of representing fuzzy class membership. First, we can "nonfuzzily" denote that a robin is a bird by defining a predicate, BIRD, that defines class membership in the set of birds and then stating that this predicate is true of robins:

or

$$
\operatorname{BIRD}(\text { robin })=T
$$$$
\operatorname{BIRD}(\text { robin })=1 \text {. }
$$

This research was supported by a grant from the Wisconsin Alumni Research Foundation, Project No. 150372. Robert Fumo assisted in conducting the experiment and Lola Lopes and Dominic Massaro provided many helpful comments and suggestions. Reprint requests should be sent to Gregg Oden, Department of Psychology, University of Wisconsin, Madison, Wisconsin 53706.
To generalize this representation to a fuzzy world only requires that one conceptualize a continuum of truth values running from absolutely true, denoted by 1.0 , to absolutely false, denoted by 0.0 . Thus, for example,

$$
\operatorname{BIRD}(\text { ostrich })=.7
$$

states that it is .7 true that an ostrich is a bird. This does not mean that $70 \%$ of all ostriches are definitely birds and $30 \%$ definitely are not. Rather, it signifies that for each ostrich it is $70 \%$ true that it is a bird.

In psychology, several recent experiments (e.g., Rips, Shoben, \& Smith, 1973; Rosch, 1973, 1975a, b) have demonstrated that the subjective degree of class membership is an important variable that affects many other cognitive processes, particularly those processes involved in answering questions by using knowledge stored in semantic memory. The class membership or superset relationship is central to most models of semantic memory (e.g., Collins \& Quillian, 1969; Kintsch, 1974; Norman \& Rumelhart, 1975), and requiring this basic relationship to be fuzzy would have far reaching effects on these important theories.

Rosch $(1973,1975 a)$ has also shown that people are adept at thinking in fuzzy terms at least in that the ratings that they give of the degree to which objects belong to a particular class are consistent both between subjects and within a given subject from one time to another. This result is very simple, but particularly important, since the use of ratings provides relatively direct information about the cognitive processes used by humans to handle fuzzy information. This is in contrast with the indirect approach of looking at these processes as they are reflected by their influence on other processes. It is, of course, generally not possible to monitor the internal psychological processes themselves directly, but the use of judgmental tasks appears to minimize the amount of intervening, extraneous processes. The present experiment continues this line of research by using a direct judgment task 
to investigate whether people can reason using fuzzy information in a consistent and systematic fashion.

The prototypical problem underlying the present experimental task is the question, "Which is more of a bird: an eagle or a pelican?" The question is both natural in style and appearance and one which is inherently concerned with fuzzy class membership. Also, the question requires a comparison of two class membership relations and, hence, must involve some amount of simple reasoning using fuzzy information. In addition, the use of two membership relations allows the construction of a factorial stimulus design which greatly increases the information provided by the experiment about the subjects' reasoning processes.

In the form given above, the question is simply one of choosing the better exemplar for the category "birds." If, in the example, the subject chooses "eagle," then additional information can be obtained about his fuzzy information processes by following the first question with "How much more of a bird is an eagle than a pelican?" In the present experiment, the questions were rephrased to make them more flexible. The subjects were asked: "Which of the following statements is truer and how much more true is it?

'An eagle is a bird.'

'A pelican is a bird.",

In this form, the task could be extended to include statements comparing degree of set membership in two different classes.

A simple model is proposed to describe the way people perform this task. The model assumes that people do, in fact, deal directly with fuzzy information to make their choice. According to the model, the person first evaluates the degree to which each statement is true and then compares the truthfulness of one statement to that of the other to determine relative truthfulness, using the following rule:

$$
R_{i j}=\frac{t\left(a_{i}\right)}{t\left(a_{i}\right)+t\left(b_{j}\right)} .
$$

For example, for the question posed above, the rule becomes:

$$
\mathrm{R}=\frac{\mathrm{BIRD}(\text { eagle })}{\mathrm{BIRD}(\text { eagle })+\mathrm{BIRD}(\text { pelican })},
$$

that is, the truth of the first statement is compared to the total truth of both statements to determine its relative truthfulness. If the two statements are varied independently in a factorial design, the relative judgment model can be tested, using the procedures of functional measurement (Anderson, 1974b).

A rule of the form of Equation 4 has been very popular as a model of probabilistic choice behavior in forced choice situations (cf. Luce, 1959). Therefore, to prevent confusion, it should be stressed that the proposed model is not at all a stochastic model, nor is it concerned with probabilities in any way. In fact, the experimental task is just the opposite of the forced choice procedure: The subject is not required to make a choice between extremes, neither of which may be considered by him to be very appropriate, but rather is explicitly allowed to respond in a continuous manner.

\section{METHOD}

Subjects were presented with stimuli consisting of two statements, each making an assertion that some item belonged to some class. For example, a representative stimulus is: $A$ chair is furniture. A robin is a bird. The subjects' task was to judge the relative truthfulness of the statements.

\section{Stimuli}

Four 5 by 5 stimulus matrices were constructed, in which the two factors of each matrix corresponded to the two statements composing each stimulus. The individual statements comprising each factor were selected to represent a wide range of degrees of category membership.

The four matrices differed in terms of the categories which were involved in the statements. The "birds" matrix consisted entirely of stimuli for which both statements asserted membership in the bird category. The "mixed-birds" matrix had one factor which was identical to one of the factors of the birds matrix and thus included only assertions about bird membership. The second factor of the mixed-birds matrix consisted of statements which were not about bird membership, but rather made assertions about membership in a mixed assortment of categories. The "furniture" and "mixed-furniture" matrices were directly analogous to the birds and mixed-birds matrices: In the former matrix, both factors were concerned solely with furniture, whereas, in the latter, one factor was concerned with furniture and one factor was concerned with a mixture of categories. The furniture factor of the mixed-furniture matrix was identical to one of the factors of the furniture matrix. The complete set of 100 stimuli can be determined by referring to Figure 1.

\section{Procedure and Apparatus}

The stimuli were typed in capital letters, one statement above the other, with the order determined randomly, on $76 \times 127 \mathrm{~mm}$ index cards. The cards were presented to the subject one at a time in random order, all matrices intermixed, at about $7 \mathrm{sec} / \mathrm{card}$. The subject stated which statement he felt to be more true and then rated the degree to which it was more true than the other. The rating response was made by placing a pin in a $200-\mathrm{mm}$ cork-topped board so that the position of the pin indicated the subject's judgment. The position of the pin was measured using a ruler attached to the back of the scale and was recorded to two decimal position accuracy as a number between 0 and 1 to signify the degree of preference for the row factor.

Subjects were first read the instructions and given about 20 stimuli for practice. This was followed by three replications of the full 100 -card deck.

\section{Subjects}

Thirty University of Wisconsin undergraduates received course credit for participating in the experiment.

\section{RESULTS}

The results of the experiment are presented in Figure 1. In this figure, the data are the circles, each of which represents the judged degree of preference for the 
row stimulus, averaged over subjects and replications. The curves are the predictions of the model when fit to the data using the iterative computer subroutine STEPIT (Chandler, 1969). This routine finds the set of parameter values which provides the best fit of the model to the data. The model was fit, using a leastsquares criterion of fit, for each subject individually, and the predictions obtained for each cell of the design were then averaged together across subjects to produce the curves shown in the figure.

As can be seen in Figure 1, the model provided an excellent fit to the data. The root mean squared deviation of the data from the model was .048 for the birds matrix, .054 for the mixed-birds matrix, .046 for the furniture matrix, and .047 for the mixedfurniture matrix. Since the computations are based on the deviations of the data from the predictions of the model for each cell for each subject, they indicate that the model provides a good fit to the data at the individual subject level, rather than merely at the group level. This is important, since group averages may be entirely unrepresentative of the individual subjects when a nonlinear process is involved, such as with the present model (Estes, 1956). Over all four matrices, the average absolute deviation of the data from the model was .039. For individual subjects, this measure ranged from .078 for the worst-fit subject to .011 for the bestfit subject. Thus, the excellent fit for every matrix provides very strong support for the model as a description of the subjects' judgmental processes.

For the purposes of fitting the model to the data, the birds matrix and the mixed-birds matrix were considered to be a single 5 by 10 matrix to reduce the number of parameters used, since the two matrices had a common factor: the column factor plotted in Figure 1. This was also done with the furniture and mixed-furniture matrices. For each such combined matrix, one of the parameters was set to 1.0 to deter-

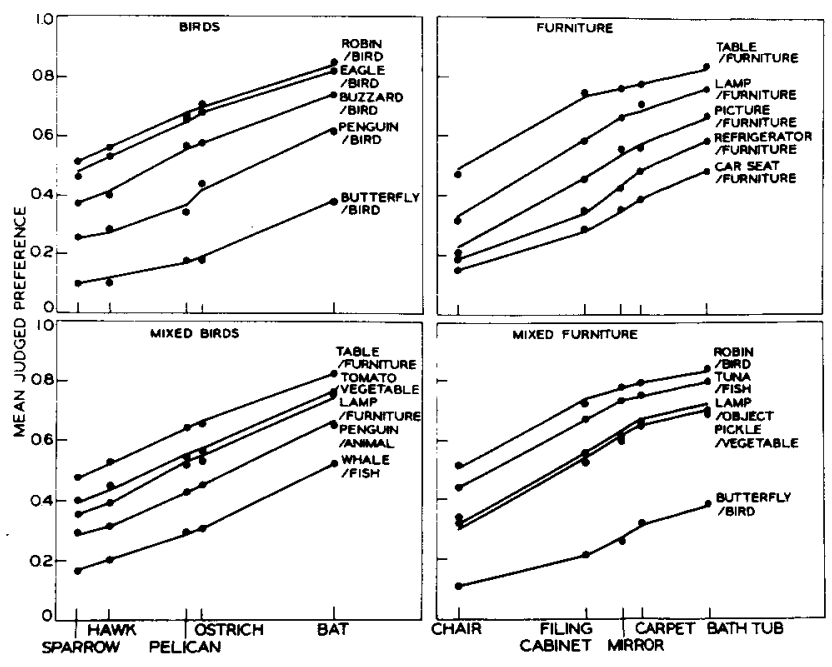

Figure 1. Mean judgments and predicted values of the truthfulness of the row statement relative to the column statement. mine the unit of the parameter scale. Thus, there were 14 model parameters, plus an additive constant to account for linear scale shifts, for a total of 15 parameters used to fit the 50 data points of each combined matrix.

In Figure 1, the spacing along the abscissa of the statements from the column factors of the matrices is proportional to the parameter values associated with those statements as determined from the fitting procedure. This is done to produce smoother curves and a more easily understandable presentation of the pattern of the interaction as described by the model. Since the birds and mixed-birds matrices were combined during the fitting of the model, they must necessarily also have the same column spacing. The fact that this produced no gross disruptions in the curves of the matrices is another indication of the overall goodness-of-fit of the model to the data. The successful use of a single set of column parameters to obtain overall goodness-of-fit for both the birds and mixed-birds matrices also indicates that the subjects were consistent in their evaluations of the truthfulness of the column statements from matrix to matrix. These facts are also true for the furniture and mixed-furniture matrices, which were similarly combined for the fitting of the model.

\section{Supplementary Statistical Analysis}

The above analysis establishes that the model provides a good description of the data. Additional analyses based on the procedure outlined by Leon and Anderson (1974) were performed to determine whether the model also provides a complete description or whether there are other systematic effects that would require a further elaboration of the model. If the model completely describes the data, then the deviations from the model should simply be random. To assess this, analyses of variance were performed on the deviations of the data from the model for each cell for each subject. These analyses are presented in Table 1 .

As can be seen in Table 1, most of the effects are not significant. This indicates that there is little that is systematic in the data that is not accounted for by the model. This is, of course, based on null effects, but the test is very powerful due to the large number of degrees of freedom. The statistical analysis indicates that, of the strong systematic effects to be accounted for in the raw data, the model does in fact account for most.

The effects in the deviations analysis which are significant are very small, especially when compared to the strong effects accounted for by the model. In fact, the ability to detect these small effects illustrates the high power of these particular statistical analyses and may increase one's confidence in interpreting the null effects as support for the model.

Furthermore, the few significant effects do not seem to follow any systematic pattern. For example, when examined in detail, neither of the significant interactions in the deviations analysis reveals any clear pattern, nor are the configurations for the two inter- 
Table 1

Analysis of Variance of the Deviations of the Data from the Model

\begin{tabular}{|c|c|c|c|c|}
\hline Source & $\mathrm{df}$ & $\mathrm{MS}_{\text {treatment }}$ & MS $_{\text {error }}$ & $\mathrm{F}$ \\
\hline \multicolumn{5}{|c|}{ Birds Matrix } \\
\hline $\begin{array}{l}\text { Mean } \\
\text { Row } \\
\text { Column } \\
\text { Interaction }\end{array}$ & $\begin{array}{r}1 \\
4 \\
4 \\
16\end{array}$ & $\begin{array}{r}193.55 \\
719.14 \\
3448.38 \\
8778.68\end{array}$ & $\begin{array}{r}192.17 \\
263.45 \\
1994.44 \\
2848.10\end{array}$ & $\begin{array}{l}1.01 \\
2.72^{*} \\
1.73 \\
3.08^{*}\end{array}$ \\
\hline \multicolumn{5}{|c|}{ Mixed-Birds Matrix } \\
\hline $\begin{array}{l}\text { Mean } \\
\text { Row } \\
\text { Column } \\
\text { Interaction }\end{array}$ & $\begin{array}{r}1 \\
4 \\
4 \\
16\end{array}$ & $\begin{array}{r}201.76 \\
101.89 \\
3120.91 \\
2717.96\end{array}$ & $\begin{array}{r}189.92 \\
282.61 \\
2008.17 \\
3997.79\end{array}$ & $\begin{array}{r}1.06 \\
.36 \\
1.55 \\
.68\end{array}$ \\
\hline \multicolumn{5}{|c|}{ Furniture Matrix } \\
\hline $\begin{array}{l}\text { Mean } \\
\text { Row } \\
\text { Column } \\
\text { Interaction }\end{array}$ & $\begin{array}{r}1 \\
4 \\
4 \\
16\end{array}$ & $\begin{array}{r}494.51 \\
451.15 \\
10997.46 \\
4978.67\end{array}$ & $\begin{array}{r}304.49 \\
308.36 \\
1541.38 \\
2601.20\end{array}$ & $\begin{array}{l}1.62 \\
1.46 \\
7.14^{*} \\
1.91 *\end{array}$ \\
\hline \multicolumn{5}{|c|}{ Mixed-Furniture Matrix } \\
\hline $\begin{array}{l}\text { Mean } \\
\text { Row } \\
\text { Column } \\
\text { Interaction }\end{array}$ & $\begin{array}{r}1 \\
4 \\
4 \\
16\end{array}$ & $\begin{array}{r}488.03 \\
1767.88 \\
9015.78 \\
2317.60\end{array}$ & $\begin{array}{r}302.66 \\
776.87 \\
1991.68 \\
2644.69\end{array}$ & $\begin{array}{l}1.61 \\
2.28 \\
4.52^{*} \\
.88\end{array}$ \\
\hline
\end{tabular}

Note-The df for error is 29 times the df for the source. Approximate critical values of $F$ at the .05 level are: for $d f=1 / 29,4.18$, for $d f=4 / 116,2.45$, and for $d f=16 / 464,1.72$.

actions similar. On the whole, then, there are a few small but statistically reliable effects in the deviations analysis which are presumably due to minor and miscellaneous cognitive processes in addition to those described by the model. They appear sporadically through the analyses and, therefore, seem to be idiosyncratic to particular stimuli, rather than reflective of the global processes involved in performing the task. In general, the model accounts for by far the majority of the trends in the data.

\section{Parameter Values}

The success of the model validates the subjects' use of the response scale according to the logic of functional measurement (Anderson, 1974b) and insures that the parameter values used to fit the model accurately reflect the subjective degree of truth of the associated statements. Since the model was fit to each subject individually, the subjective truth values were also separately obtained for each individual. Table 2 presents the subjective truth values for the group which are the geometric means of the individual subject values. Geometric means were used since, in the present model, it is the ratio of parameters to each other, not the difference between them, which determines their effect on the response. As described above, the parameter for $A$ robin is a bird, which was judged to be most true, was set to 1.0 to determine the unit of the subjective scale. Therefore, these values must strictly be interpreted as only proportional to the actual subjective values. However, since for most people a robin is probably considered to be nearly absolutely a bird, the other values are also probably near the actual values.

The parameter values largely follow prior expectations. A couple, however, do deviate somewhat from expectation and are, therefore, particularly interesting. For example, it had been assumed that the statement $A$ penguin is an animal would be rated as almost completely true, on the reasoning that, to the extent that a penguin is not a bird, it must be a mammal or a fish, and, in any event, certainly an animal. Further questioning of subjects revealed, however, that many consider the term "animal" to be more synonymous with "mammal" than with "animate being." Thus, the parameter values reflect the fact that a penguin is subjectively "sort of a bird" and "sort of a mammal."

Another, more fundamental, result is that for each category there is a wide range of things which are considered to be good members of that category. For example, both tables and chairs were judged to be very good exemplars of furniture, even though tables and chairs are fairly semantically dissimilar considering their size, shape, function, and so on. This has implications for theories of category structure which will be discussed below.

\section{DISCUSSION}

The results of the present experiment support the notion that humans are competent processors of fuzzy information. The subjects in this study used their knowledge about the degree of membership in the class of birds, furniture, and so on to perform their complex judgment task in a consistent and systematic fashion. That the subjects were using the fuzzy information very consistently is a fundamental implication of the success of the relative judgment model, since the model used each of its parameters to make predictions about several different responses. For example, the model was able to account for the responses of each subject to 10 different stimuli for which the statement $A$ sparrow is a bird was a component, while using only a single value for the parameter representing the subjective degree to which a sparrow belongs to the class of birds. This success, therefore, requires that the subjects also had a consistent, uniform conception of the "birdiness" of sparrows that they used in solving each of the judgmental problems, including "mixed" as well as "pure"

Table 2

Parameter Values: The Degree of Class Membership

\begin{tabular}{lrlrll}
\hline \multicolumn{2}{c}{ Birds } & \multicolumn{2}{c}{ Furniture } & \multicolumn{1}{c}{ Other } \\
\hline robin & 1.000 & chair & .960 & tuna (fish) & .667 \\
sparrow & .997 & table & .855 & tomato (vegetable) & .546 \\
eagle & .836 & lamp & .358 & penguin (animal) & .251 \\
hawk & .769 & picture & .119 & lamp (object) & .213 \\
buzzard & .494 & filing cabinet & .114 & pickle (vegetable) & .200 \\
pelican & .420 & mirror & .091 & whale (fish) & .117 \\
ostrich & .345 & carpet & .057 & & \\
penguin & .233 & refrigerator & .053 & & \\
bat & .125 & bath tub & .032 & & \\
butterfly & .019 & car seat & .030 & & \\
\hline
\end{tabular}


problems. This is an important and fundamental result, but one which is often overlooked when evaluating the significance of the success of such models.

The success of the model with the mixed-birds and mixed-furniture matrices is particularly important in indicating that the subjects were reasoning directly in terms of fuzzy class membership in performing the task. It had been originally suspected that people might have difficulty handling the problem of a "mixed" stimulus. With the other, "pure" matrices, one is not forced to initially determine the degree of truth of each individual statement. Rather, it is possible to conceive of strategies for these cases in which one might attempt to compare the candidate class members directly to each other before referring to the class itself. With the mixed stimuli, no such shortcut strategy appears to be possible. Therefore, the fact that the subjects were able to successfully handle the more complex problems indicates a competency for reasoning directly with fuzzy information.

\section{Validity of the Information About \\ Fuzzy Knowledge Provided by \\ Subjective Judgments}

The present study also verifies the fact established by previous studies (Rips et al., 1973; Rosch, 1973, 1975a) that people have conscious access to the results of much of their fuzzy information processing. For situations where this is true, the rating procedure used here provides direct and detailed information about the subjects' fuzziness competency. Much of this information is very difficult, if at all possible, to obtain by other means. Psychologists, however, are sometimes suspicious of subjects' ratings, in part because of the feeling that the subjects might take into consideration irrelevant information when making their judgments and that, therefore, the ratings will not accurately reflect the underlying processes of interest. While this may occasionally be true, it is also sometimes the case that the subjects know more about what is relevant to the processes of interest than psychologists do. For example, Rips et al. (1973) felt that it might be the case that the important information in their reaction time tasks would be the similarity of various birds and mammals to the classes of "birds," "mammals," and "animals" within a euclidian multidimensional representation of these concepts. However, when multidimensional scaling procedures were used to filter out all of the nonmultidimensional information from the subjects' ratings, they were less successful in accounting for the reaction time data than when the raw ratings were used. Thus, the subjects' intuitions about class membership, as reflected in their ratings, included information in addition to the multidimensional information, and the additional information was apparently very important in performing the reaction time tasks.

Other psychologists are sometimes suspicious of ratings because they are not confident that subjects will use the rating scale appropriately. If the ratings are not a linear function of the subjects' subjective values, then the pattern of the data may be misleading with respect to the underlying processes which produced it. The use of functional measurement (Anderson, 1974b) in the present study eliminates this problem through the power of factorial designs and a quantitative model: The model could not have been successful in accounting for the data if subjects had misused the response scale unless the model were actually incorrect and the subjects had misused the scale in exactly the manner required to cancel out the deficiencies of the model. This becomes even less of a reasonable alternative explanation as more and more studies find subjects using the rating scale properly.

The validation of the response scale by the success of the model insures that the obtained parameter values are direct measures of the actual subjective degree of class membership values. The parameter values should be highly stable estimates of the subjective values, since each is based on the judgments made by the subjects to several different problems. Thus, on theoretical grounds, one can have quite a bit more confidence in these values than in previously obtained estimates of the subjective values.

It is interesting to compare the present values with those obtained in previous studies. For example, 17 of the statements used in the present study correspond directly with items also used by Rosch (1975a). In her study, subjects simply rated the degree to which things belonged to particular classes using a 7-point discrete category scale on which a rating of 7 signified that the item did not belong to the class at all. Note that this scale runs in the opposite direction from the present parameter scale, on which high values signify a high degree of membership. The mean ratings from Rosch's study are plotted in Figure 2 as a function of the parameter values from the present study for the 17 common terms. As is clear from the figure, there is a fairly strong degree of correspondence between the values from the two studies. In fact, the -.95 correlation between the two sets of values is rather large considering

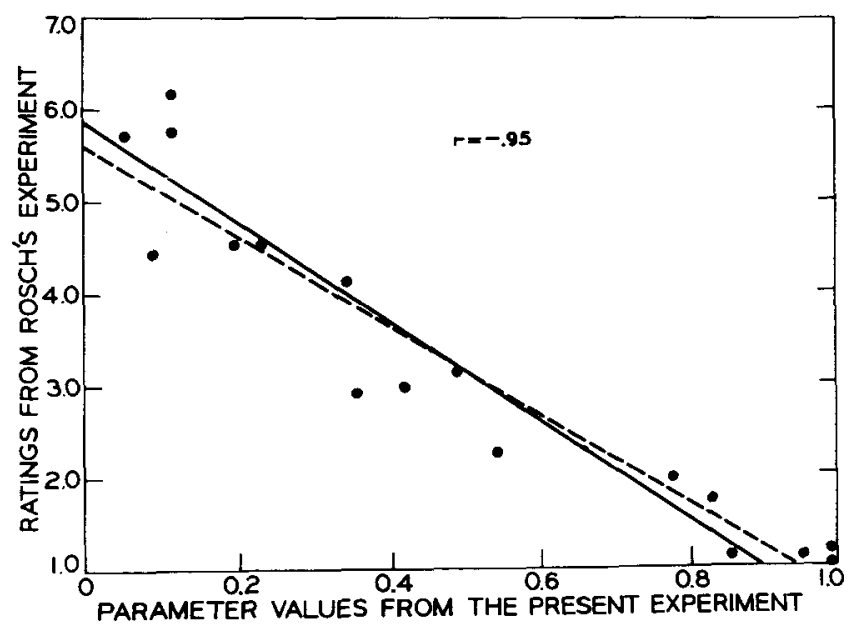

Figure 2. Subjective degree of class membership values from Rosch's (1975a) experiment plotted as a function of the values obtained in the present experiment. The lines show the regression of each set of values on the other. 
that this is a comparison between different experiments conducted at different places and times. However, it also seems clear from Figure 2 that the deviations from linearity are fairly systematic: The points tend to fall below the regression lines toward the middle of the scale and above the lines toward the ends of the scale. This curvilinearity is not surprising, since simple category rating procedures such as that used by Rosch tend to introduce some degree of nonlinearity (Anderson, 1974a). However, Rosch's values were certainly precise enough for the purposes of her experiment, and the fact that the functional measurement procedures used here produced values which are more exact is not the important point to be drawn from the comparison. Instead, what should be emphasized is that subjective class membership values are quite stable and consistent across groups of subjects and that ratings can provide rich and reliable information about this kind of knowledge.

\section{Internal Structure of Semantic Categories}

Once it is agreed that category membership is a matter of degree, the question naturally arises as to how categories are internally structured, that is, what determines the degree of membership for each exemplar? One common hypothesis (e.g., Rosch, 1973) is that there is a prototype for each category and the degree of membership for each item is directly related to the similarity of the item to the prototype. Given this general prototype notion, the pattern of the parameter values of the present experiments is quite interesting. Specifically, for both birds and furniture, there seems to be a diverse set of items which are judged to be quite good members. For example, tables and chairs do not seem to be highly similar, yet both are judged to be quite good exemplars of furniture. Similarly, eagles and robins differ in size, habitat, shape, diet, beauty of song, and ferocity, among other things, but both are considered to be good birds. Furthermore, while eagles and buzzards would seem to have about as much in common as do eagles and robins, buzzards are judged to be considerably less good examplars of birds.

The pattern of parameter values is not inconsistent with the general prototype notion but does restrict it to some extent. There are at least three possible restricted prototype hypotheses which could be compatible with the present results: (1) The degree of class membership is not proportional to degree of similarity to the prototype. Rather, there is a roughly ogival function relating class membership to similarity with a greater slope in the middle of the range than at the ends. (2) Degree of class membership is proportional to similarity, but the prototype lies somewhere between robins and hawks for birds, and between chairs and tables for furniture. (3) Categories may have multiple prototypes.

Each of the more restricted hypotheses has its merits. The first hypothesis seems to be natural for other fuzzy concepts such as "tall" or "close to 5 " and is what is often implicitly assumed (e.g., Lakoff, 1972; Zadeh, 1975). The second hypothesis has received some measure of empirical support from the multidimensional scaling data of Rips et al. (1973), in which the point for "bird" fell between "robin" and "eagle."

However, a severe disadvantage of both of these hypotheses is that they make the strongly nonintuitive prediction that modifying a perfect table to make it more similar to a chair (e.g., by adding a back and padding) should make it an even better exemplar of furniture. Only the third hypothesis can escape from making this prediction. It does so by having separate chair-furniture and table-furniture prototypes. Similarly, for the bird category, the hypothesis would allow both a song-bird prototype and a bird-of-prey prototype, each of which would signify very good kinds of birds. This approach has considerable intuitive appeal, but, clearly, more data will be necessary to decide among the alternative hypotheses.

\section{Impact of Fuzziness Competency on Semantic Models}

Treating class membership as a discrete all-or-none relation has been very convenient in developing models of semantic memory and semantic information processing. In the beginning stages of model construction, such oversimplifications are typically necessary to keep the whole problem within manageable limits. However, as models mature, convenient oversimplifications usually become neither convenient nor simplifying: The attempt to fit more and more sophisticated phenomena within an overly weak theoretical framework generally results in complex and often grossly contorted descriptions.

In this light, it would appear to be a very healthy sign that many of the important models of semantic memory have now incorporated mechanisms for representing fuzzy semantic information (e.g., Carbonell \& Collins, 1973; Kintsch, 1974; Norman \& Rumelhart, 1975 ) and that inference and retrieval processes have also been proposed which depend on and capitalize upon the fuzzy information (e.g., Carbonell \& Collins, 1973; Collins \& Loftus, 1975). This is a very important beginning, since higher level semantic processes will build upon the fundamental structures and processes.

One particularly important higher level semantic process is the use of semantic constraints or semantic selectional restrictions in language to determine whether or not a sentence is sensible. Semantic constraints are restrictions on the possible meanings of different parts of sentences: The sentence will be sensible if its parts are semantically compatible (cf. Katz \& Fodor, 1963). But since the meanings of most of the sentence parts will include various fuzzy aspects, it seems much more reasonable to talk about the degree to which two sentence parts are semantically compatible. This leads naturally to considering that the degree to which a sentence is sensible will be determined by the degree to which its semantic constraints are satisfied. Oden 
and Anderson (1974) found subjects to make judgments about the sensibleness of sentences using processes of this sort.

Semantic constraints provide important information which can be used by other stages of language processing, for example, in speech perception (Reddy, Erman, \& Neely, 1973; Woods \& Makhoul, 1973) and in syntactic analysis (Schank, 1972; Winograd, 1973). Language comprehension is a complex problem and is likely to require intensive use of the various kinds of information available to the comprehender, including the continuous information derived from semantic constraints. In fact, Oden (Note 1) has shown that there are many ambiguous sentences which cannot be properly disambiguated unless the information provided by the fuzziness of semantic constraints is used.

As the illustrations suggest, the human competency for processing fuzzy information can be expected to permeate virtually every cognitive process that uses semantic information. Rather than requiring a separate stage in our human information processing model, it will probably necessitate elaboration of many of the components. Hopefully, the result will be a less fictional, more realistic description of human cognition.

\section{REFERENCE NOTE}

1. Oden, G. C. On the use of semantic constraints in guiding syntactic analysis. Unpublished manuscript.

\section{REFERENCES}

Anderson, N. H. Algebraic models in perception. In E. C. Carterette \& M. P. Friedman (Eds.) Handbook of perception (Vol. 2). New York: Academic Press, 1974. (a)

ANDERson, N. H. Information integration theory: A brief survey. In D. H. Krantz, R. C. Atkinson, R. D. Luce, \& P. Suppes (Eds.) Contemporary developments in mathematical psychology (Vol. 2). San Francisco: W. H. Freeman, 1974. (b)

Carboneli, J. R., \& Collins, A. N. Natural semantics in artificial intelligence. Proceedings of the Third International Joint Conference on Artificial Intelligence, 1973, 344-351.

Chandler, J. P. Subroutine STEPIT-Finds local minima of a smooth function of several parameters. Behavioral Science, 1969, 14, 81-82.

Collins, A. M., \& Loftus, E. R. A spreading-activation theory of semantic processing. Psychological Review, 1975, 82, 407-428.

Collins, A. M., \& Quillian, M. R. Retrieval time from semantic memory. Joumal of Verbal Leaming and Verbal Behavior, 1969, 8. 240-247.

Estes, W. K. The problem of inference from curves based on group data. Psychological Bulletin, 1956, 53, 134-140.

KATZ, J. J., \& Fodor, J. A. The structure of a semantic theory. Language, 1963, 39, 170-210.

KinTSCH. W. The representation of meaning in memory. New York: Halsted, 1974.

LAKOFF, G. HEDGES: A study in meaning criteria and the logic of fuzzy concepts. Papers from the eighth regional meeting of the Chicago Linguistic Society. Chicago: University of Chicago Linguistic Department, 1972.

Leon, M., \& Anderson, N. H. A ratio role from integration theory applied to inference judgments. Journal of Experimental Psychology, 1974, 102, 27-36.

LuCE, R. D. Individual choice behavior. New York: Wiley, 1959.

Norman, D. A., \& Rumelhart, D. E. Explorations in cognition. San Francisco: W. H. Freeman, 1975.

Oden, G. C., \& ANDERSON, N. H. Integration of semantic constraints. Journal of Verbal Learning and Verbal Behavior, 1974, 13, 138-148.

Reddy, D. R., Erman, L. D., \& Neely, R. B. A model and a system for machine recognition of speech. IEEE Transaction on Audio and Electroacoustics, 1973, AU-21, 229-238.

Rips, L. J., Shoben, E. J., \& S Mith. E. E. Semantic distance and the verification of semantic relations. Journal of Verbal Learning and Verbal Behavior, 1973, 12, 1-20.

Rosch, E. H. On the internal structure of semantic and perceptual categories. In T. E. Moore (Ed.) Cognitive development and the acquisition of language. New York: Academic Press, 1973

Rosch, E. H. Cognitive representations of semantic categories. Journal of Experimental Psychology: General, 1975, 104, 192-233. (a)

Rosch, E. H. The nature of mental codes for color categories. Journal of Experimental Psychology: Human Perception and Performance, 1975. 1, 303-322. (b)

Schank, R. C. Conceptual dependency: A theory of natural language understanding. Cognitive Psychology, 1972, 3, $552-631$.

Winograd, T. A procedural model of language understanding. In R. C. Shank \& R. M. Colby (Eds.) Computer models of thought and language. San Francisco: W. H. Freeman, 1973.

Woods, 'W. A., \& MAKHoul, J. Mechanical inference problems in continuous speech understanding. Proceedings of the Third International Joint Conference on Artificial Intelligence, 1973, 200-207.

ZADEH, L. Fuzzy sets. Information and Control, 1965, 8, 338-353.

ZADEH, L. A. Calculus of fuzzy restrictions. In L. A. Zadeh, K. S. Fu, K. Tanaka, \& M. Shimura (Eds.) Fuzzy sets and their applications to cognitive and decision processes. New York: Academic Press, 1975.

(Received for publication May 28, 1976; revision received September 13, 1976.) 